\title{
An Area-to-Inv Bijection Between Dyck Paths and 312-avoiding Permutations
}

\author{
Jason Bandlow and Kendra Killpatrick
}

Mathematics Department

Colorado State University

Fort Collins, Colorado

bandlow@math.colostate.edu

killpatr@math.colostate.edu

Submitted: July 23, 2001; Accepted: December 10, 2001.

MR Subject Classifications: 05A15, 05A19

\begin{abstract}
The symmetric $q, t$-Catalan polynomial $C_{n}(q, t)$, which specializes to the Catalan polynomial $C_{n}(q)$ when $t=1$, was defined by Garsia and Haiman in 1994. In 2000, Garsia and Haglund described statistics $a(\pi)$ and $b(\pi)$ on Dyck paths such that $C_{n}(q, t)=\sum_{\pi} q^{a(\pi)} t^{b(\pi)}$ where the sum is over all $n \times n$ Dyck paths. Specializing $t=1$ gives the Catalan polynomial $C_{n}(q)$ defined by Carlitz and Riordan and further studied by Carlitz. Specializing both $t=1$ and $q=1$ gives the usual Catalan number $C_{n}$. The Catalan number $C_{n}$ is known to count the number of $n \times n$ Dyck paths and the number of 312-avoiding permutations in $S_{n}$, as well as at least 64 other combinatorial objects. In this paper, we define a bijection between Dyck paths and 312-avoiding permutations which takes the area statistic $a(\pi)$ on Dyck paths to the inversion statistic on 312-avoiding permutations. The inversion statistic can be thought of as the number of (21) patterns in a permutation $\sigma$. We give a characterization for the number of $(321),(4321), \ldots,(k \cdots 21)$ patterns that occur in $\sigma$ in terms of the corresponding Dyck path.
\end{abstract}

\section{Introduction}

The polynomial $C_{n}(q, t)$, called the $q, t$-Catalan polynomial, was introduced in 1994 by Garsia and Haiman [5]. They conjectured that it is the Hilbert series of the diagonal harmonic alternates and showed that it is the coefficient of the elementary symmetric function $e_{n}$ in the symmetric polynomial $D H_{n}(x ; q, t)$; the conjectured Frobenius characteristic of the module of diagonal harmonic polynomials. The polynomial is referred to as the $q, t$-Catalan polynomial because specializing $t=1$ gives the $q$-Catalan polynomial 
first given by Carlitz and Riordan [3] and further studied by Carlitz [2]. Specializing both $q=t=1$ results in the well-known Catalan number $C_{n}=\frac{1}{n+1}\left(\begin{array}{c}2 n \\ n\end{array}\right)$.

In order to give the precise definition of $C_{n}(q, t)$, we must first introduce some notation. A sequence $\mu=\left(\mu_{1}, \mu_{2}, \ldots, \mu_{k}\right)$ is said to be a partition of $n$ if $\mu_{1} \geq \mu_{2} \geq \cdots \geq \mu_{k}>0$ and $\mu_{1}+\mu_{2}+\cdots+\mu_{k}=n$. A partition $\mu$ may be described pictorially by it's Ferrers diagram, an array of $n$ dots into $k$ left-justified rows with row $i$ containing $\mu_{i}$ dots for $1 \leq i \leq k$. Using the Ferrers diagram we can define the transpose of a partition $\mu$, denoted $\mu^{T}$, to be the partition whose $i$ th row (numbered from the top) is the length of the $i$ th column in the Ferrers diagram of $\mu$. The symbols $l$ and $l^{\prime}$ are used to represent the leg and coleg of a cell: the number of cells strictly below and strictly above a given cell, respectively. Similarly, $a$ and $a^{\prime}$ represent the arm and coarm of a cell: the number of cells strictly to the right and strictly to the left of a given cell, respectively. For example, for the labelled cell $s$ in the diagram below, $a=5, a^{\prime}=4, l=3$, and $l^{\prime}=2$.

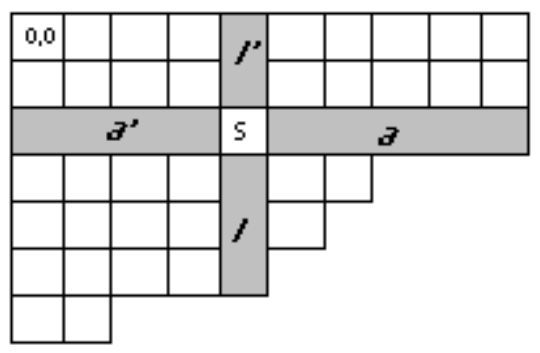

The precise definition of $C_{n}(q, t)$ is given as follows:

$$
C_{n}(q, t)=\sum_{\mu \vdash n} \frac{t^{2 \Sigma l} q^{2 \Sigma a}(1-t)(1-q) \prod^{(0,0)}\left(1-q^{a^{\prime}} t^{l^{\prime}}\right) \sum q^{a^{\prime}} t^{l^{\prime}}}{\prod\left(q^{a}-t^{l+1}\right)\left(t^{l}-q^{a+1}\right)}
$$

The summations and products within the $\mu^{\text {th }}$ summand are over all cells in the Ferrers diagram of a given partition, and the symbol $\Pi^{(0,0)}$ is used to represent the product over all cells but the upper left corner.

The $q, t$-Catalan polynomial is symmetric in $q$ and $t$; i.e, $C_{n}(q, t)=C_{n}(t, q)$. To see this, note that for every $\mu \vdash n, \mu^{T}$ is also a partition of $n$. We can see that the summand corresponding to $\mu$ in $C_{n}(q, t)$ will equal the summand corresponding to $\mu^{T}$ in $C_{n}(t, q)$ by observing the relationships between $a, l, a^{\prime}$, and $l^{\prime}$ in $\mu$ and $\mu^{T}$. Given a cell $s$ in a partition $\mu$, the arm length of $s$ in $\mu$ equals the leg length of the corresponding cell $s^{\prime}$ in $\mu^{T}$, and vice-versa. Similarly, the lengths of the coarm and coleg of $s$ and $s^{\prime}$ are also interchanged. This can be seen in the diagram below, which shows the transpose of the first diagram, and the corresponding cell, $s^{\prime}$. Note that here, $l=5, l^{\prime}=4, a=3$, and $a^{\prime}=2$. 




Though symmetry follows directly from the definition of the $q, t$-Catalan polynomial, it is less obvious that this polynomial has positive integer coefficients. To prove this, Garsia and Haiman conjectured that there exist statistics $a(\pi)$ and $b(\pi)$ on $n \times n$ Dyck paths, $D_{n}$, such that $C_{n}(q, t)=\sum_{\pi \in D_{n}} q^{a(\pi)} t^{b(\pi)}$. (A complete description of Dyck paths follows in Section 2.) For a given path $\pi$, the statistic $a(\pi)$ is the number of full squares which lie below the path and completely above the line $y=x[4]$. To compute $b(\pi)$, we first construct a second Dyck path from $\pi$ called $\beta(\pi)$; then $b(\pi)$ is the sum of the $x$-coordinates of the points where this second path touches the line $y=x$, excluding the points $(0,0)$ and $(n, n)$. (See Section 3 for a more complete description of $b(\pi)$.) The $b(\pi)$ statistic was first conjectured by Haglund [7] and then proved to be correct by Garsia and Haglund [5].

Since $C_{n}(q, t)=C_{n}(t, q)$, there must exist a bijection from $D_{n}$ to $D_{n}$ that maps $\pi$ to $\pi^{\prime}$, such that $a(\pi)=b\left(\pi^{\prime}\right)$ and $b(\pi)=a\left(\pi^{\prime}\right)$. It is an open problem to define such a bijection constructively. A possible approach to find such a bijection is to make use of some of the 66 other known descriptions of the Catalan numbers [11]. More work has been done on the development of statistics for some of these objects than others.

In particular, many statistics have been developed for permutations. While investigating permutations that could be sorted on a single pass through a stack, Knuth [8] showed that (312)-avoiding permutations satisfy the Catalan recurrence. The inv statistic, or inversion statistic, is defined as the number of "inversions" in a permutation, or alternately as $n(21)$ : the number of patterns of the form (21) in a permutation. Fürlinger and Hofbauer [4] proved that the $q$-Catalan polynomial is the generating function for inversions on (312)-avoiding permutations.

The main result of this paper is to give a bijection from Dyck paths to (312)-avoiding permutations that sends the area statistic on Dyck paths to the inv statistic on (312)avoiding permutations. In addition, we will classify the occurrence of permutation patterns $n(321), n(4321), \ldots, n(k \cdots 21)$ in terms of Dyck paths.

Since the $a(\pi)$ and $b(\pi)$ statistics are equidistributed on Dyck paths, the hope is to find another statistic on permutations which, when restricted to (312)-avoiding permutations, gives the corresponding $b(\pi)$ statistic under our bijection. An examination of the statistics known to be equidistributed with inv, however, has not yet yielded any such result. 
In section 2, we give the necessary definitions and background for this paper. Section 3 contains the construction of our bijection and the proof that it sends the area statistic on Dyck paths to the inv statistic on (312)-avoiding permutations. Section 4 gives our characterization of $n(321), n(4321), \ldots, n(k \cdots 21)$, and Section 5 discusses some open questions.

\section{Background and Definitions}

The Catalan sequence is the sequence

$$
\left\{C_{n}\right\}_{n=0}^{\infty}=\{1,1,2,5,14,42, \ldots\}
$$

where

$$
C_{n}=\frac{1}{n+1}\left(\begin{array}{c}
2 n \\
n
\end{array}\right)
$$

$C_{n}$ is called the $n$th Catalan number. The Catalan numbers have been shown to count certain properties on more than 66 different combinatorial objects (see Stanley [11] pg. 219, Exercise 6.19 for a complete list). The objects of use to us in this paper will be certain lattice paths called Dyck paths and certain permutations called 312-avoiding permutations.

A Dyck path is a lattice path in $\mathbb{Z}^{2}$ from $(0,0)$ to $(n, n)$ consisting of only steps in the positive $x$ direction (EAST steps) and steps in the positive $y$ direction (NORTH steps) such that there are no points $(x, y)$ on the path for which $x>y$. In other words, a Dyck path is a path from $(0,0)$ to $(n, n)$ consisting only of NORTH and EAST steps that never goes below the diagonal. Let $D_{n}$ denote the set of Dyck paths from $(0,0)$ to $(n, n)$. For example, $D_{3}$ consists of the following paths:

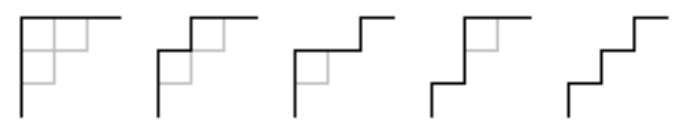

The Catalan number $C_{n}$ is known to count the number of Dyck paths from $(0,0)$ to $(n, n)$, thus $C_{3}=5$. The length of a Dyck path is the number of NORTH steps in the path, thus a Dyck path $\pi \in D_{n}$ has length $n$.

Let $S_{n}$ denote the symmetric group on $[n]=\{1,2, \ldots, n\}$. A transposition $s_{i}=(i, i+1)$ is a function from $S_{n}$ to $S_{n}$ which interchanges the numbers in the $i$ th and $(i+1)$ st position in a permutation. For example,

$$
s_{4}(512867394)=512687394 .
$$

It is well-known that every permutation $\sigma$ in $S_{n}$ can be represented as a sequence of transpositions $s_{i_{1}} s_{i_{2}} \ldots s_{i_{k}}$ which, when applied from right to left to the identity permutation $123 \cdots n$, results in $\sigma$. This representation is not necessarily unique. For example, 321 can be written as both $s_{1} s_{2} s_{1}$ and $s_{2} s_{1} s_{2}$. We will describe one method for writing a permutation as such a product of transpositions in the following section. 
A 312-avoiding permutation $\pi \in S_{n}$ is a permutation $\pi=\pi_{1} \pi_{2} \cdots \pi_{n}$ containing no triple $\pi_{i} \pi_{j} \pi_{k}$ with $i<j<k$ such that $\pi_{i}>\pi_{k}>\pi_{j}$. For example, $\pi=2143$ is a (312)-avoiding permutation in $S_{4}$ while $\sigma=4213$ is not.

Let $S_{n}(312)$ denote the set of all 312-avoiding permutations in $S_{n}$ and let $A_{n}(312)=$ $\left|S_{n}(312)\right|$. In [8], Knuth proved that, for every $R \in S_{3}$,

$$
A_{n}(R)=\frac{1}{n+1}\left(\begin{array}{c}
2 n \\
n
\end{array}\right)=C_{n}
$$

In particular, if $R=(312)$, this proves that $C_{n}$ equals the number of 312-avoiding permutations.

In addition to having an explicit formula, the Catalan numbers are known to satisfy the recurrence

$$
C_{n}=\sum_{i=1}^{n} C_{i-1} C_{n-i} .
$$

This can easily be visualized using the Dyck paths. Given a Dyck path from $(0,0)$ to $(n, n)$, label the diagonal points in $\mathbb{Z}^{2}$ as $a_{i}=(i, i)$ for $1 \leq i \leq n$. Let

$$
A_{i}=\left\{\text { Dyck paths from }(0,0) \text { to }(n, n) \text { that first touch the diagonal at } a_{i}\right\} .
$$

In other words, $A_{i}$ is the set of paths for which $i$ is the smallest integer such that $(i, i)$ is a point on the path. Then clearly $C_{n}=\sum_{i=1}^{n}\left|A_{i}\right|$. It remains to show that $\left|A_{i}\right|=C_{i-1} C_{n-i}$.

If a path first touches the diagonal at $(i, i)$, the path must go from $(0,1)$ to $(i-1, i)$ without touching the diagonal points $(1,1),(2,2), \ldots,(i-1, i-1)$. The number of such paths is $C_{i-1}$. Once the path touches $(i, i)$ it must then continue to $(n, n)$ without going below or to the right of the diagonal. The number of such paths is $C_{n-i}$. Thus $\left|A_{i}\right|=C_{i-1} C_{n-i}$ and therefore

$$
C_{n}=\sum_{i=1}^{n}\left|A_{i}\right|=\sum_{i=1}^{n} C_{i-1} C_{n-i}
$$

For example, if $n=10$ and $i=3$, then any path in $A_{3}$ must go from $(0,0)$ to $(0,1)$, then take some path from $(0,1)$ to $(2,3)$ without touching $(1,1)$ or $(2,2)$. Since the chosen path is in $A_{3}$, it must then go from $(2,3)$ to $(3,3)$ and then it can take any valid Dyck path from $(3,3)$ to $(10,10)$. One example of such a path is: 


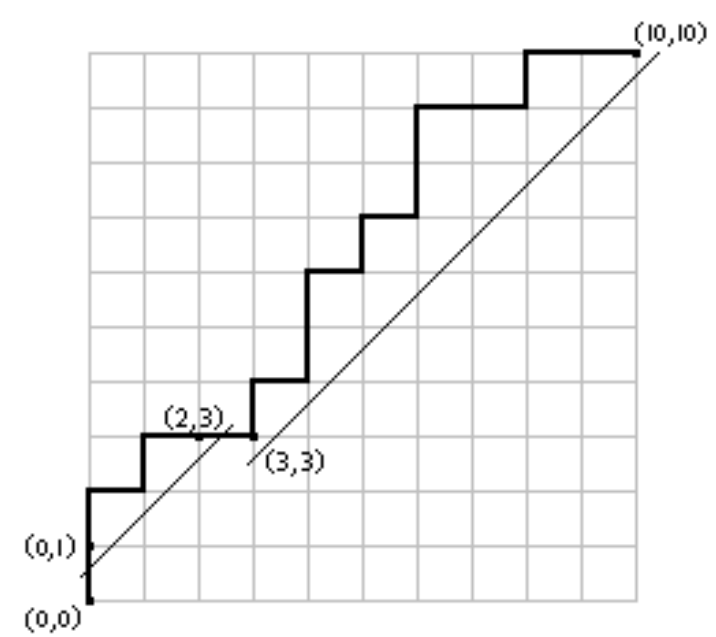

A statistic on a permutation, Dyck path, or other combinatorial object counts some property about that object. The inversion statistic on a permutation $\sigma \in S_{n}$ is defined by

$$
\operatorname{inv}(\sigma)=\sum_{\substack{1 \leq i<j \leq n \\ \sigma_{i}>\sigma_{j}}} 1 .
$$

For example, if $\sigma=743216598$, then $i n v(\sigma)=14$ since each of the pairs (21), (31), (41), (71), (32), (42), (72), (43), (73), (74), (65), (75), (76), and (98) contributes 1 to the sum.

The generating function for the inversion statistic on $S_{n}$ is given by

$$
\sum_{\sigma \in S_{n}} q^{i n v(\sigma)} .
$$

Two different statistics on a class of objects are said to be equidistributed if they have the same generating function on that class of objects. A statistic on permutations is called Mahonian if it is equidistributed with the inv statistic on permutations in $S_{n}$. One wellknown Mahonian statistic is the major index, written $\operatorname{maj}(\sigma)$, first given by MacMahon [10]. The major index is defined in terms of descents in a permutation. A descent in a permutation $\sigma=\sigma_{1} \sigma_{2} \ldots \sigma_{n}$ is a position where $\sigma_{i}>\sigma_{i+1}$. For example, $\sigma=7136254$ has 3 descents. The major index is defined as the sum of the positions of the descents of $\sigma$, i.e.

$$
\operatorname{maj}(\sigma)=\sum_{\sigma_{i}>\sigma_{i+1}} i
$$

For the previous permutation $\sigma, \operatorname{maj}(\sigma)=1+4+6=11$.

In addition to defining statistics on permutations, we can define statistics on Dyck paths. Given a Dyck path $\pi \in D_{n}$ the area statistic, $a(\pi)$, is the number of squares that lie below the path and completely above the diagonal. For example, given the following Dyck path the squares counted by the area statistic are shaded, giving an area statistic of 13 . 


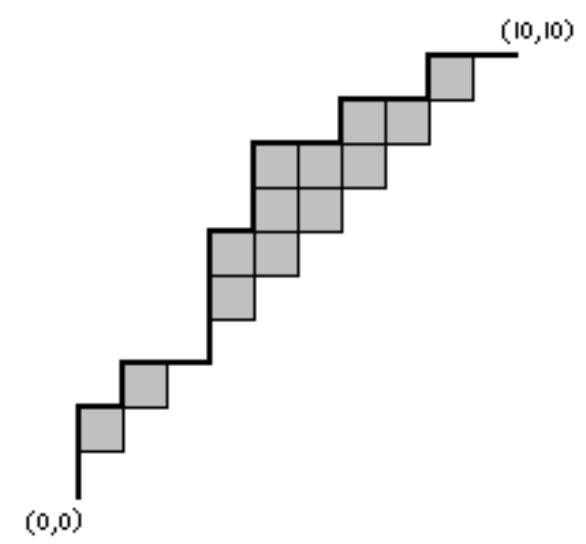

The generating function for the area statistic on Dyck paths $\pi \in D_{n}$,

$$
\sum_{\pi \in D_{n}} q^{a(\pi)}=C_{n}(q)
$$

is the $q$-Catalan polynomial [4]. Specializing $q=1$ in the $q$-Catalan polynomial gives the usual Catalan number $C_{n}$. Fürlinger and Hofbauer [4] showed that

$$
C_{n}(q)=\sum_{i=1}^{n} q^{i-1} C_{i-1}(q) C_{n-i}(q) .
$$

To visualize this recurrence, use notation similar to our explanation of the recurrence for the Catalan numbers. Let

$$
A_{i}(q)=\sum_{\pi \in A_{i}} q^{a(\pi)}
$$

Clearly,

$$
C_{n}(q)=\sum_{i=1}^{n} A_{i}(q) .
$$

Then to understand the $q$-Catalan recurrence, it is necessary to understand why

$$
A_{i}(q)=q^{i-1} C_{i-1}(q) C_{n-i}(q) .
$$

Since a path in $A_{i}$ first touches the diagonal at $(i, i)$, it must go from $(0,1)$ to $(i-1, i)$ without touching the diagonal points $(1,1),(2,2), \ldots,(i-1, i-1)$. These number of such paths has been shown to be $C_{i-1}$ and thus have weight $C_{i-1}(q)$. To these paths, we must add the $i-1$ squares just above the diagonal from $(0,0)$ to $(i, i)$. Thus the part of the paths from $(0,0)$ to $(i, i)$ in $A_{i}$ give us a weight of $q^{i-1} C_{i-1}(q)$. From $(i, i)$, the paths must then continue on to $(n, n)$ without going below the diagonal. These paths have weight $C_{n-i}(q)$, giving us a total weight of

$$
A_{i}(q)=q^{i-1} C_{i-1}(q) C_{n-i}(q) .
$$

Using the same example of a path in $A_{3}$ as previously, the additional 2 squares giving the weight $q^{2}$ are shaded in black: 


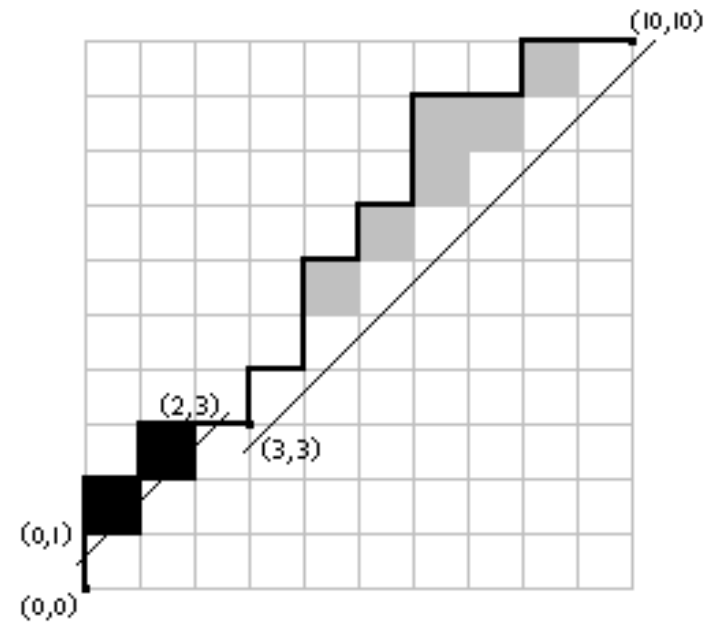

Returning to the q,t-Catalan polynomial of Garsia and Haiman [6], these authors showed that $C_{n}(q, 1)=C_{n}(q)$. In addition, they conjectured the existence of a statistic $b(\pi)$ on Dyck paths $\pi \in D_{n}$ such that

$$
C_{n}(q, t)=\sum_{\pi \in D_{n}} q^{a(\pi)} t^{b(\pi)} .
$$

Haglund [7] conjectured that $b(\pi)$ was given by a statistic he called $\operatorname{maj}(\beta(\pi))$. This conjecture was recently proved by Garsia and Haglund [5].

To determine the statistic $\operatorname{maj}(\beta(\pi))$ for $\pi \in D_{n}$, one first obtains the path $\beta(\pi)$ which can be thought of as a "billiard ball" path. To obtain this path from $\pi \in D_{n}$, first imagine shooting a ball straight WEST from $(n, n)$ and just below the path until reaching a vertical step in $\pi$. Reflect the path of the ball directly SOUTH from this point until reaching the diagonal. At the diagonal, reflect the path directly WEST and still slightly under the path $\pi$ until reaching another vertical step in $\pi$, upon which the path is again reflected SOUTH until reaching the diagonal. Continue in this manner until reaching the point $(0,0)$.

Label the diagonal points by $(i, i)=n-i$ for $1 \leq i \leq n-1$. Then $b(\pi)=\operatorname{maj}(\beta(\pi))$ is the sum of the labels where the path $\beta(\pi)$ touches the diagonal (not including $(n, n)$ or $(0,0))$. For example, the bold line denotes the path $\pi \in D_{n}$ and the dashed line denotes the path $\beta(\pi)$ in the diagram below. 


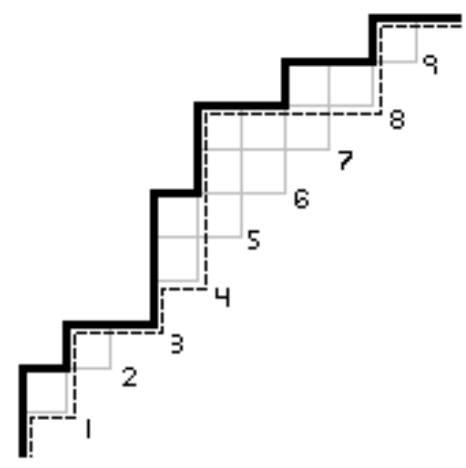

For this path $\pi, b(\pi)=\operatorname{maj}(\beta(\pi))=2+6+7+9=24$.

By the symmetry of $C_{n}(q, t)$, as explained in the Introduction section,

$$
C_{n}(q, t)=C_{n}(t, q)
$$

So

$$
C_{n}(q)=C_{n}(q, 1)=C_{n}(1, q)=C_{n}(1, t)=C_{n}(t, 1)=C_{n}(t)
$$

Thus

$$
\sum_{\pi \in D_{n}} q^{a(\pi)}=\sum_{\pi \in D_{n}} t^{b(\pi)}
$$

While a bijection between Dyck paths is known [9] that sends a Dyck path $\pi_{1}$ to a Dyck path $\pi_{2}$ such that $b\left(\pi_{1}\right)=a\left(\pi_{2}\right)$, this bijection does not have the property that $a\left(\pi_{1}\right)=b\left(\pi_{2}\right)$. Finding such a bijection is an interesting open problem and would give a combinatorial proof of the symmetry of the $q, t$-Catalan polynomial.

\section{Bijection Between Dyck Paths and 312-avoiding Permutations}

Before stating and proving our main theorem, we will describe a well-defined method for writing a permutation $\sigma \in S_{n}(312)$ as a product of adjacent transpositions $s_{i}$.

Let $\sigma \in S_{n}(312)$. Write $\sigma$ as a product of adjacent transpositions $s_{i}$ by first determining a specific sequence of adjacent transpositions which, when applied to $\sigma$, will give the identity permutation. Then $\sigma$ can be represented by the inverse of this sequence of transpositions.

To determine the specific sequence of adjacent transpositions, suppose $n$ is in position $i$ in $\sigma$. Then $s_{n-1} s_{n-2} \cdots s_{i+1} s_{i}$ (applied right to left) moves the $n$ to position $n$ and leaves the relative order of the numbers 1 through $n-1$ unchanged. Now locate $n-$ 1 in the resulting permutation. Suppose $n-1$ is in position $j$. Then the sequence $s_{n-2} s_{n-3} \cdots s_{j+1} s_{j}$ moves the $n-1$ to position $n-1$. Continuing in this manner will give the identity permutation. Then $\sigma$ can be represented as the inverse of this sequence of transpositions. Since $s_{i}^{2}=i d$ then $s_{i}^{-1}=s_{i}$ so the inverse of this sequence of transpositions is the same sequence written in reverse order. Thus $\sigma$ is represented by a product of 
adjacent transpositions $s_{i}$ whose subscripts form a series of increasing subsequences, i.e., $\sigma=\sigma_{1} \sigma_{2} \cdots \sigma_{j}$ with $j \leq n$ such that each $\sigma_{i}$ is a product of adjacent transpositions whose subscripts are strictly increasing. In this representation, $j$ is the minimum number of such subsequences.

For example, let

$$
\sigma=\begin{array}{llllllllll}
2 & 3 & 1 & 6 & 8 & 7 & 9 & 5 & 10 & 4 .
\end{array}
$$

Then $s_{9}$ moves the 10 to the last position, giving

$$
s_{9}(\sigma)=\begin{array}{lllllllllll}
2 & 3 & 1 & 6 & 8 & 7 & 9 & 5 & 4 & 10 .
\end{array}
$$

Next $s_{8} s_{7}$ moves the 9 to the 9 th position, $s_{7} s_{6} s_{5}$ moves the 8 to the 8th position, $s_{6} s_{5}$ moves the 7 to the 7 th position, $s_{5} s_{4}$ moves the 6 to the 6 th position, $s_{4}$ moves the 5 to the 5 th position, the 4 is already in the 4 th position, $s_{2}$ moves the 3 to the 3 rd position, and $s_{1}$ moves the 2 to the 2 nd position. Then $\sigma$ can be represented as the inverse of this sequence of transpositions, so

$$
\sigma=s_{9} / s_{7} s_{8} / s_{5} s_{6} s_{7} / s_{5} s_{6} / s_{4} s_{5} / s_{4} / s_{2} / s_{1} .
$$

The symbol / has been added above only as a delimiter for the sake of readability.

In this example, $\sigma=\sigma_{1} \sigma_{2} \cdots \sigma_{8}$ where $\sigma_{1}=s_{9}, \sigma_{2}=s_{7} s_{8}, \sigma_{3}=s_{5} s_{6} s_{7}, \sigma_{4}=s_{5} s_{6}$, $\sigma_{5}=s_{4} s_{5}, \sigma_{6}=s_{4}, \sigma_{7}=s_{2}$, and $\sigma_{8}=s_{1}$.

Now we describe a function $f: S_{n}(312) \rightarrow D_{n}$.

Let $\sigma=\sigma_{1} \sigma_{2} \cdots \sigma_{k}$, where each $\sigma_{i}$ is a subsequence of adjacent transpositions with increasing subscripts, using the method described.

For each $i$, if $\sigma_{i}$ has length $l$ and ends with $s_{m}$, then shade in the squares of $\mathbb{Z}^{2}$ in the $(m+1)$ st row and in columns $m$ through $m-l+1$. Then $f(\sigma)$ is the Dyck path that has these shaded squares and only these shaded squares below it. Note that no Dyck path will ever have squares in the first row or $n$th column since all Dyck paths must start with a NORTH step and end with an EAST step.

For $\sigma=\sigma_{1} \sigma_{2} \ldots \sigma_{8}=s_{9} / s_{7} s_{8} / s_{5} s_{6} s_{7} / s_{5} s_{6} / s_{4} s_{5} / s_{4} / s_{2} / s_{1}$, as in the previous example, then $f(\sigma)$ is the following Dyck path:



THe ELECtronic Journal of COMBINATORICs 8 (2001), \#R40 
To find $f^{-1}$ of a Dyck path, shade in the squares below the path. Then read rows from top to bottom and within each row read left to right, writing down an $s_{j}$ for a shaded square in column $j$.

Lemma 1. If $\pi$ is a Dyck path, then $f^{-1}(\pi)$ is a 312-avoiding permutation.

Proof. Proof by induction on $n$, the length of the Dyck path.

Suppose $n=1$. There is only one Dyck path consisting of a north step and then an east step. This path bijects to the identity permutation $\sigma=1$ which is clearly 312-avoiding.

Assume that if $\pi$ is a Dyck path of length $n-1$, then $f^{-1}(\pi)$ is a 312-avoiding permutation in $S_{n-1}$. Let $\hat{\pi}$ be a Dyck path of length $n$. If there are no squares under the path in the top row, then $f^{-1}$ maps $\hat{\pi}$ to a permutation with $n$ in the $n$th position. In this case, it is enough to check that the permutation in positions 1 through $n-1$ is 312 avoiding. By induction, the path from $(0,0)$ to $(n-1, n-1)$ bijects to a permutation in $S_{n-1}$ and is 312-avoiding, thus adding $n$ to the end still gives a 312-avoiding permutation.

Suppose there exist squares under the Dyck path $\hat{\pi}$ in row $n$ and in columns $j$ through $n-1$. In order for $\hat{\pi}$ to be a Dyck path, there must be squares under the Dyck path in row $n-1$ and columns $j$ through $n-2$. If $\hat{\pi}$ is a Dyck path of length $n$, then the shaded squares under the path in the first $n-1$ rows form a Dyck path of length $n-1$ which by induction bijects to a 312-avoiding permutation. Let $\sigma$ denote this permutation of length $n-1$. Thus it remains to check that $s_{j} s_{j+1} \cdots s_{n-1}(\sigma \mathbf{n})$ is 312-avoiding.

This sequence of $s_{i}$ 's applied to $\sigma \mathbf{n}$ moves the $n$ to the left by interchanging it with smaller numbers. Since $\sigma$ was a 312 -avoiding permutation, $s_{j} s_{j+1} \cdots s_{n-1}(\sigma \mathbf{n})$ could only fail to be a 312-avoiding permutation if the $n$ moves two or more positions to the left of the $n-1$. However, if $n-1$ is in position $i$ of $\sigma$ then the Dyck path has shaded squares in row $n-1$ in columns $i$ through $n-1$, so in row $n$ shaded squares could only lie in column $i$ or columns to the right. Thus $j \geq i$ and so $s_{j} s_{j+1} \cdots s_{n-1}(\sigma \mathbf{n})$ moves $n$ to position $j$ which is either to the right of position $i$ or which equals position $i$. If $j>i$, then $n$ remains to the right of $n-1$ and so the resulting permutation is 312-avoiding. If $j=i$ then $s_{j}$ interchanges $n$ and $n-1$ so $n$ is one position to the left of $n-1$ and the resulting permutation is 312 -avoiding.

Lemma 2. If $\sigma$ is a 312-avoiding permutation, then $f(\sigma)$ is a Dyck path.

Proof. Proof by induction on $n$. If $n=1$, then the only permutation is $\sigma=1$ and the resulting path consists of one north and then one east step, which is a valid Dyck path.

Now assume that for every $\sigma \in S_{n-1}(312), f(\sigma)$ is a Dyck path. Let $\sigma \in S_{n}$ be a 312-avoiding permutation. Let $\tau \in S_{n-1}$ be the permutation in $S_{n-1}$ such that $s_{j} s_{j+1} \cdots s_{n-1}(\tau \mathbf{n})=\sigma$. Since the sequence $s_{j} s_{j+1} \cdots s_{n-1}$ affects only the relative position of $n$ in the permutation, if $\sigma$ is 312-avoiding then $\tau$ is also 312-avoiding. By induction $f(\tau)$ is a Dyck path. Suppose $n-1$ is in position $i$ in $\tau$, then there are squares under the Dyck path in row $n-1$ in columns $i$ through $n-1$. Since $\sigma$ is 312 -avoiding, the position of $n$ in $\sigma$ is either to the right of the position of $n-1$ in $\sigma$ or one position to the left of $n-1$. Thus $j \geq i$ and so in the Dyck path every shaded square in row $n$ lies above a 
shaded square in row $n-1$. Since $f(\tau)$ was a Dyck path of length $n-1$ by induction, then $f(\sigma)$ is a Dyck path of length $n$.

Lemma 3. If $\sigma$ has $k$ inversions then when $\sigma$ is written as a product of adjacent transpositions $s_{i}$ as described, $\sigma$ has exactly $k$ terms in the product. I.e., every $s_{i}$ in the representation of $\sigma$ corresponds to an inversion in $\sigma$.

Proof. Suppose $\sigma=\sigma_{1} \sigma_{2} \cdots \sigma_{k}$ when written as a product of transpositions in the manner described, with each $\sigma_{i}$ equal to a product of transpositions with increasing subscripts. Suppose $\sigma_{i}=s_{j} s_{j+1} \cdots s_{l}$. Then this sequence of transpositions interchanges the position of $l$ with the element to the left of $l$ which by construction is always less than $l$, so each swap introduces exactly one inversion. Similarly, if $(l k)$ is an inversion in $\sigma$, then there must exist an $s_{m}$ in the representation of $\sigma$ that interchanges $l$ and $k$. Otherwise $l$ would remain to the right of $k$ and $(l k)$ would not be an inversion in $\sigma$.

Theorem 1. The function $f$ is a weight-preserving bijection from $S_{n}(312)$ to $D_{n}$ that maps the inversion statistic to the area statistic.

Proof. From Lemmas 1 and 2 we may conclude that $f$ is a bijection from 312-avoiding permutations to Dyck paths and from Lemma 3 it follows directly that $f$ maps a 312avoiding permutation with $k$ inversions to a Dyck path with area statistic $k$.

\section{Classifications of other permutation patterns}

The number of inversions in a permutation $\sigma$ may also be described as the number of (21) patterns that appear in $\sigma$, i.e., as the number of pairs $(i, j)$ with $i<j$ such that $\sigma_{i}>\sigma_{j}$. Denote the number of (21) patterns in $\sigma$ as $n_{\sigma}(21)$. In the previous section, we show that $n_{\sigma}(21)$ equals the area statistic on $f(\sigma)$. Generalizing this result, $n_{\sigma}(321)$, $n_{\sigma}(4321), \ldots, n_{\sigma}(k \cdots 21)$ can all be described in terms of the Dyck path $f(\sigma)$ and can be determined using only $f(\sigma)$ by counting patterns called symmetric patterns. Define a symmetric pattern of size 3 as a pattern on an $n \times n$ grid of the form



such that squares $a_{11}$ and $a_{12}$ lie in the same row but not necessarily in adjacent columns and such that squares $a_{11}$ and $a_{21}$ lie in the same column but not necessarily in adjacent rows. By symmetric, we mean that if there are $k$ columns between $a_{11}$ and $a_{12}$, then there are $k$ rows between $a_{11}$ and $a_{21}$. In general, a symmetric pattern of size $k$ is a pattern on an $n \times n$ grid of the form 


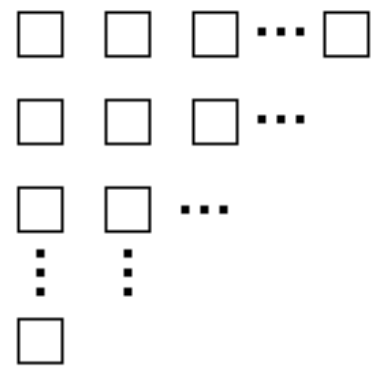

with $k-1$ squares in the first row and column of the pattern, $k-2$ squares in the second row and column of the pattern, etc. The squares in the second row of the pattern must lie in the same columns as the first $k-2$ squares in the first row. The squares in the third row of the pattern must lie in the same columns as the first $k-3$ squares in the first row, etc. In this more general example, symmetric means that if there are $k$ columns between $a_{i j}$ and $a_{i m}$, then there are $k$ rows between $a_{j i}$ and $a_{m i}$. Since the patterns are symmetric, they are completely determined by the position of the squares in the top row.

Lemma 4. For any 312-avoiding permutation $\sigma$, the number $n_{\sigma}(321)$ equals the number of symmetric patterns of size 3 under the Dyck path $f(\sigma)$. In general $n_{\sigma}(k \cdots 21)$ is the number of symmetric patterns of size $k$ that lie under the Dyck path $f(\sigma)$. Algebraically,

$$
n_{\sigma}(k \cdots 21)=\sum_{\begin{array}{c}
\text { rows in the } \\
\text { Dyck path } f(\sigma)
\end{array}}\left(\begin{array}{c}
\text { area }(\text { row }) \\
k-1
\end{array}\right)
$$

where area(row) is the number of squares under the Dyck path in that row.

Proof. First note that if $s$ is a square under a given Dyck path $\pi$, then every square above the diagonal that lies below or to the right of $s$ is also a square under the Dyck path $\pi$.

Suppose $i_{1}<i_{2}<\cdots<i_{k-1}<m$ and $\left(m i_{k-1} \ldots i_{2} i_{1}\right)$ is a pattern contained in $\sigma$. Let $P_{m, k}$ be the set of decreasing patterns of length $k$ in $\sigma$ that begin with $m$ and let $I_{m, k}$ be the set of numbers, not including $m$, that appear in the patterns in $P_{m, k}$.

Order and label the elements in $I_{m, k}$ as $i_{1}, i_{2}, \ldots, i_{j}$ such that $i_{1}<i_{2}<\cdots<i_{j}$. Then there are at least $j$ squares under the Dyck path $f(\sigma)$ in row $m$ since each inversion with $m$ corresponds to a square under the Dyck path in row $m$.

Lemma 5. If $P_{m, k} \neq \emptyset$, then there are exactly $j$ squares in row $m$.

Proof. Suppose there were at least $j+1$ squares in row $m$. Then in $\sigma, m$ is part of at least $j+1$ inversions. The $\left(m i_{1}\right),\left(m i_{2}\right), \cdots,\left(m i_{j}\right)$ account for $j$ inversions so there exists an $l \notin I_{m, k}$ such that $(m l)$ is an inversion in $\sigma$. Since $i_{1}<i_{2}<\cdots<i_{j}<m$ and they all form inversions with $m$, they must appear in decreasing order in $\sigma$, otherwise $\sigma$ would contain a 312 pattern. If $l>i_{j}$ then the pattern $\left(m l i_{j} i_{j-1} \ldots i_{j-(k-3)}\right)$ must appear in $\sigma$, otherwise $\sigma$ would contain a 312 pattern. But then $l$ would be an element of $I_{m, k}$ which gives a contradiction. Now suppose $i_{q}<l<i_{q+1}$. If $q<(k-2)$ then the pattern $\left(m i_{k-2} \cdots i_{q+1} l i_{q} \cdots i_{1}\right)$ must appear in $\sigma$, otherwise $\sigma$ would contain a 312 
pattern. If $q \geq(k-2)$ then the pattern $\left(m l i_{q} i_{q-1} \cdots i_{q-(k-3)}\right)$ appears in $\sigma$. In both of these cases, $l$ would then be an element of $I_{m, k}$, giving a contradiction. Finally, if $l<i_{1}$ then $\left(m i_{k-2} \cdots i_{1} l\right)$ must appear in $\sigma$, otherwise $\sigma$ contains a 312 pattern and thus $l$ would be in $I_{m, k}$. Again, this gives a contradiction. Thus there are exactly $j$ squares in row $m$.

Choose any $k-1$ elements from $I_{m, k}$. These $k-1$ elements, when put together with $m$, correspond uniquely to a $(k \cdots 21)$ pattern in $\sigma$. In addition, these $k-1$ elements each correspond uniquely to a square under the Dyck path in row $m$, since each element forms an inversion with $m$, thus this set of $k-1$ squares determines a unique symmetric pattern of size $k$ as previously described, with $k-1$ squares in row $m$. Then summing over all rows under the Dyck path gives all possible symmetric patterns of length $k$, i.e.

$$
n_{\sigma}(k \cdots 21)=\sum_{\substack{\text { rows in the } \\
\text { Dyck path } f(\sigma)}}\left(\begin{array}{c}
\text { area(rows) } \\
k-1
\end{array}\right) .
$$

For example, if $\sigma=43521$, the Dyck path $f(\sigma)$ is:

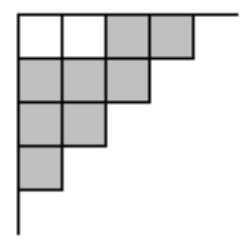

This permutation $\sigma$ contains the (321) patterns (321), (421), (431), (432), and (521) which correspond respectively to the following symmetric patterns of size 3 :
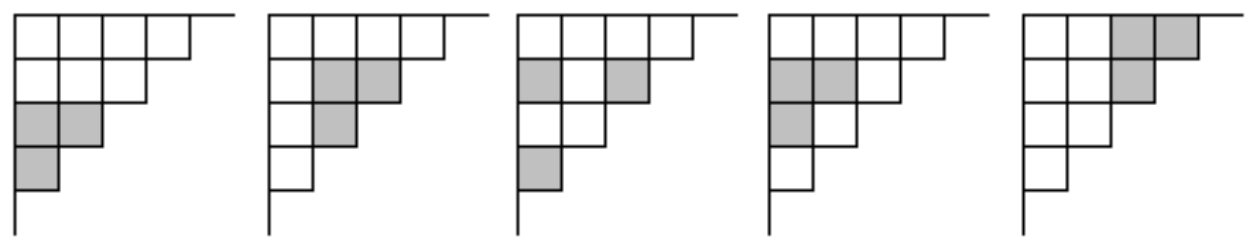

In general, for an $\left(m i_{k-1} \cdots i_{2} i_{1}\right)$ pattern in $\sigma, m$ determines the row and $i_{k-1}, \ldots, i_{2}, i_{1}$ determine which squares in that row form the symmetric pattern of size $k$. 


\section{Open Problems}

A combinatorial proof of the symmetry of the $q, t$-Catalan polynomial, in which one bijects a Dyck path $\pi_{1}$ to a Dyck path $\pi_{2}$ such that $a\left(\pi_{1}\right)=b\left(\pi_{2}\right)$ and $b\left(\pi_{1}\right)=a\left(\pi_{2}\right)$ remains elusive. Our approach is to translate this problem from Dyck paths to permutations, where the study of statistics is more developed. This paper translates the area statistic on Dyck paths to inv on 312-avoiding permutations. The hope of the authors is to find an easily defined statistic on 312-avoiding permutations which gives the $b(\pi)$ statistic, and then use the ideas of known permutation bijections to prove symmetry. One approach to finding a nice description of the $b(\pi)$ statistic is to study Mahonian statistics, which are by definition equidistributed with inv on all of $S_{n}$, and determine if any restrict to the proper statistic on 312-avoiding permutations. However, Babson and Steingrimsson [1] recently classified essentially all known Mahonian permutation statistics in terms of the occurrence of patterns of length at most 3 (such as 321 and 213 patterns) and none of these statistics properly restrict to the $b(\pi)$ statistic on 312 -avoiding permutations. Thus this approach must involve finding a new Mahonian statistic which restricts properly on 312-avoiding permutations, or simply finding a new statistic which is either only defined on 312-avoiding permutations or is not Mahonian on all permutations.

\section{Acknowledgements}

The authors would especially like to thank Eric Egge for his many helpful comments and suggestions. We look forward to future collaborations.

THE ELECTRONic JOURNAL OF COMBINATORICs 8 (2001), \#R40 


\section{References}

[1] BABSON, E. and STEINGRIMSSON, E. Generalized permutation patterns and a classification of the Mahonian statistics. Sem. Lothar. Combin. 44 (2000), Art. B44b, 18pp.

[2] CARLITZ, L. Sequences, paths, ballot numbers. Fibonacci Quarterly 10 (1972), 531-549.

[3] CARLITZ, L. and RIORDAN, J. Two element lattice permutation numbers and their q-generalization. Duke J. Math 31 (1964) 371-388.

[4] FÜRLINGER, J. and HOFBAUER, J. q-Catalan Numbers. Journal of Combinatorial Theory, Series A 40 (1985) 248-264.

[5] GARSIA, A. and HAGLUND, J. A Proof of the $q$, t-Catalan Positivity Conjecture. Preprint. (2000).

[6] GARSIA, A. and HAIMAN, M. A remarkable $q$, t-Catalan sequence and $q$-Lagrange inversion. J. Algebraic Combinatorics 5 (1996), no. 3, 191-244.

[7] HAGLUND, J. Conjectured Statistics for the $q, t$-Catalan Numbers. Preprint. (2000).

[8] KNUTH, D.E. The Art of Computer Programming, Volume 1, Addison-Wesley, Reading, MA 1973.

[9] ANDREWS, G., KRATTENTHALER, C., ORSINA, L., and PAPI, P. ad-Nilpotent b-ideals in $s l(n)$ having a fixed class of nilpotence: Combinatorics and enumeration. Trans. Amer. Math. Soc. (to appear).

[10] MACMAHON, P.A. Combinatory Analysis, Vols. I, II, Chelsea, New York, 1984.

[11] STANLEY, R. Enumerative Combinatorics, Volume 2, Cambridge University Press, 1999.

THE ELECTRONic JOURNAL OF COMBINATORICs 8 (2001), \#R40 